\title{
Prophylactic antibiotics to prevent surgical site infections in Botswana; findings and implications
}

\author{
Julius C. Mwita ${ }^{1}$, Sajini Souda², Mgaywa GMD Magafu ${ }^{3}$, Amos Massele $^{4}$, Brian Godman ${ }^{5,6,7^{*}}$, Michael \\ Mwandri ${ }^{8}$ \\ 1Department of Internal Medicine, Faculty of Medicine, University of Botswana, Gaborone, Botswana. \\ Email: mwitajc@ub.ac.bw \\ 2Department of Pathology, Faculty of Medicine, University of Botswana, Gaborone, Botswana. Email: \\ sajinisouda@gmail.com \\ ${ }^{3}$ Department of Global Health, University of Washington, Blantyre, Malawi. Email: \\ gmdmgaywa@yahoo.com \\ ${ }^{4}$ Department of Biomedical Sciences, Faculty of Medicine, University of Botswana, Gaborone, \\ Botswana. Email: amos.massele@mopipi.ub.bw \\ ${ }^{5}$ Division of Clinical Pharmacology, Karolinska Institute, Karolinska University Hospital Huddinge, \\ Stockholm, Sweden. Email: Brian.Godman@ki.se \\ ${ }^{6}$ Strathclyde Institute of Pharmacy and Biomedical Sciences, Strathclyde University, Glasgow, UK. \\ Email: Brian.Godman@strath.ac.uk \\ ${ }^{7}$ Health Economics Centre, Liverpool University Management School, Liverpool, UK. Email: \\ Brian.Godman@liverpool.ac.uk \\ ${ }^{8}$ School of Clinical Medicine, University of KwaZulu-Natal, Durban, South Africa. Email: \\ mwanrister@gmail.com
}

*Corresponding author - Brian Godman - Strathclyde Institute of Pharmacy and Biomedical Sciences, University of Strathclyde, Glasgow, United Kingdom. Email: Brian.godman@strath.ac.uk and Division of Clinical Pharmacology, Karolinska Institute, Karolinska University Hospital Huddinge, Stockholm, Sweden. Email: Brian.Godman@ki.se.

Key words: surgical site infection; antibiotic prophylaxis; timing of prophylaxis; organisms; Botswana

(Accepted for publication in Hospital Practice Journal - please keep Confidential)

\begin{abstract}
Background and aims: Antibiotic prophylaxis in surgery is known to reduce the rate of surgical site infections (SSI) as well as shorten hospital stay. However, there is currently a scarcity of data on antibiotic prophylaxis and SSIs among African countries including Botswana. Consequently, this study aimed to address this. Methods: A prospective study involving 400 patients was carried out at a leading tertiary hospital in Botswana from 2014-2015. Patients' demographic information, type of surgery performed and peri-operative use of antibiotics were documented. All enrolled patients were followed-up for 30 days post discharge to fully document the incidence of SSIs. Results: Median age of patients was $35.5(25-50)$ years, with $52 \%$ female. There were $35.8 \%$ emergency and $64.2 \%$ elective surgeries. The most common operations were exploratory laparotomy (25\%), appendectomy $(18.3 \%)$, excision and mastectomy $(8 \%)$. Antibiotics were given in $73.3 \%$ of patients, mainly postoperatively $(58.3 \%)$. The most commonly prescribed antibiotics were cefotaxime $(80.7 \%)$, metronidazole $(63.5 \%)$, cefradine $(13.6 \%)$ and amoxicillin/clavulanate $(11.6 \%)$. The incidence of SSI was $9 \%$. The most common organisms were Pseudomonas aeruginosa, Staphylococcus aureus, and coagulase-negative staphylococci. Conclusion: The rate of SSI is a concern, and may be related to inappropriate antibiotic prophylaxis given post operatively. Interventions are in place to decrease SSI rates to acceptable levels in this leading hospital by improving for instance infection prevention practices including the timing of antibiotic prophylaxis. Research is also ongoing among other hospitals in Botswana to reduce SSI rates building on these findings.
\end{abstract}

\section{Introduction}

Surgical site infections (SSI) are infections related to the operative procedure that occurs at or near the surgical incision within 30 days of the procedure (up to one year if a prosthetic material is implanted), and affecting either the incision or deep tissue at the operation site or any part of the anatomy (e.g., organs and spaces) [1-3]. They are among the common causes of hospital acquired infections, which appreciably add to post-operative morbidity and mortality, prolong hospital stay by 
up to 7 to 11 post-operative days, and increase costs [3-5]. Patients with SSIs can increase their mortality by up to 11 -fold, with SSI costing up to US $\$ 10 \mathrm{bn} /$ year across countries $[5,6]$. The incidence of SSI ranges from under $1 \%$ to over $50 \%$ depending on population studied, type of surgery, size of hospital, ventilation within the hospital, experience of the surgeon and surveillance method [3, 7-10]. In a meta-analysis, Ngaroua et al reported a pooled incidence rate of SSI of $14.8 \%$ in Africa [11], with SSIs appearing particularly problematic and common in lower and middle income countries (LMICs) versus high income countries [12].

The pattern of occurrence of SSI is based on the distribution and virulence of the offending organisms and host resistance [13]. Common causative organisms of SSI include staphylococcus species, Escherichia coli, Pseudomonas aeruginosa and Klebsiella species [10, 14-16]. Polymicrobial existence is common in SSIs $[15,16]$. Risk factors for development of SSI include the degree of tissue trauma, pre-operative infection, presence of a prosthesis or other foreign body, surgical technique, length of surgery and its complexity, re-interventions, reduced fitness, use of steroids, lack of adherence to agreed prescribing guidance and a prolonged duration of surgery [10, 13, 17-19].

Over the years, surgery has been made safer by modifying these factors through measures such as aseptic surgical techniques, skin cleansing prior to surgery, pre-operative patient preparation, optimization of co-morbid illnesses and by administering surgical antibiotic prophylaxis $[13,20]$. Surgical antibiotic prophylaxis reduces the burden of microorganisms at the surgical site and has been shown to be a cost-effective strategy in reducing the rate of SSI and shortening hospital stay $[13,21,22]$. Overall, up to $60 \%$ of SSIs are preventable using evidence based approaches [6]. The use of antibiotics has been shown to decrease SSI by $39 \%$ [23]. Guidelines recommend that antibiotic prophylaxis be given within 1-2 hours before incision, and in doses sufficient to achieve adequate serum and tissue drug levels for the interval during which the surgical site is open $[6,17$, $20,22,24-26]$. In cases where the duration of the surgery exceeds 2 half-lives of the antimicrobial or there is excessive blood loss, intraoperative re-dosing is needed to maintain adequate serum and tissue drug levels [27]. However, initiating antibiotics after the first incision is not advised as it almost doubles SSI rates [28]. Moreover, prolongation of antibiotic prophylaxis after the operation is not recommended as it predisposes to resistance $[26,29]$. Continued surveillance and appropriate strategies can appreciably reduce the prevalence of SSIs, which include the monitoring of clinical practice against agreed guidance [30]. Such strategies are becoming increasingly important.

Antibiotic selection is often based on the risk of wound infection according to wound classification, the normal floral distribution of the site to be operated and the antibiotic resistance patterns in the hospital setting $[1,6,17,25,27,31,32]$. Generally, broad-spectrum antibiotics are preferred to narrow spectrum antibiotics and cephalosporin are commonly used [27]. Cefazolin is commonly recommended for gastroenterological, genito-urinary tract, and hepatobiliary surgeries [27]. The Botswana national guidelines recommend cefradine for cardiothoracic, neurosurgery, gastroenterological, vascular surgeries, breast, orthopaedic and gynaecological surgeries [33]. A combination of ampicillin, gentamicin and metronidazole is recommended for high risk biliary surgery, and a combination of gentamicin and ciprofloxacin for urological surgeries [33]. Despite these recommendations, studies have shown inconsistencies in administering surgical prophylaxis in for instance the proportion of surgical patients to whom prophylaxis is prescribed, type of the antibiotic chosen, time of administration and duration of the antibiotic course [17, 26]. In addition, there is paucity of information regarding the burden of SSI and prophylactic regimens used in developing countries; although, as mentioned, these are likely to be more problematic in LMICs $[7,12]$.

\section{Aims}

We sought to address the lack of knowledge regarding SSIs in Botswana by describing their current burden and the antibiotic surgical prophylactic patterns in a leading hospital in Botswana. The findings would act as a basis for developing future pertinent strategies to reduce the number of SSIs in this hospital and wider in Botswana. This is particularly important in Botswana with its high incidence of infectious diseases, e.g. $21.9 \%$ of the adult population currently have HIV although this rate is decreasing [34]. 


\section{Methodology}

\section{Study setting and participants}

This prospective cross-sectional study was conducted at Princess Marina Hospital (PMH) in Gaborone, between February 2014 and June 2015. PMH is the main public hospital in the country with a 560-bed capacity and an average of 12 general surgical operations every day. Consequently, if there were concerns with surgical prophylaxis in this hospital, such concerns or worse are highly likely at secondary hospitals in Botswana. We only included adult patients who underwent surgery, resided within a perimeter of 50 kilometres from PMH and with whom home visits were possible in view of our follow-up regime. We excluded children in this first study as adults are the largest population as well as patients with prostheses or implants.

\section{Data collection}

The information obtained from all enrolled patients included: demographic characteristics, anthropometric measurements, surgical diagnoses, comorbid conditions and the duration of antibiotics use. Other information included the type and time of prophylactic antibiotic administration. Enrolled patients were followed up for 30 days after the surgical procedure to assess the development of SSI. While in the wards, patients were assessed for the development of signs and symptoms of SSI once every two days until hospital discharge. We assessed for fever $\left(>38^{\circ} \mathrm{C}\right)$, abnormal wound discharge, increasing pain or tenderness, redness, heat or localized swelling/ abscess. Radiological, histological and clinical diagnoses of wound infection made by the attending doctor were also documented.

After discharge, patients were physically assessed for SSIs through home visits or an arranged hospital visit during the first week post-discharge the hospital. Thereafter, patients were contacted through telephone calls twice every week until the $30^{\text {th }}$ day post discharge to determine the development of any SSIs. During the phone calls, patients were asked if they had any symptoms of a SSI as described above. For those with any of the SSI symptoms, we arranged physical examination and pus swabs for microbiological examination. The diagnosis of an SSI was made on the presence of one or more of the following: purulent exudate from a surgical site, positive fluid culture - from a surgical site that was closed primarily; reopened surgical site due to at least one clinical sign of infection (pain, swelling, erythema, warmth) - culture positive or not cultured; or surgeon's diagnosis of infection. Patients with SSIs were referred to the responsible unit at PMH where they were managed accordingly.

\section{Statistical analysis}

The sample size was calculated from the assumption that the incidence of SSIs in Botswana was $15 \%$, and an expected loss to follow up of $30 \%$ [11]. We needed 400 patients to determine the true incidence with a margin of error of $\pm 4 \%$ using a $95 \%$ confidence interval. Data were analysed using SPSS Version 23.0 for Windows (SPSS Inc., Chicago, IL, USA). Normally distributed variables were expressed as mean \pm standard deviation (SD) and asymmetrically distributed variables were presented as median [interquartile range (IQR)]. We present categorical and nominal variables as absolute and relative frequencies (\%).The Pearson's $\mathrm{X} 2$ test was used to contrast categorical data. We considered a two sided $p$ value of $<0.05$ to be statistically significant.

\section{Ethical consideration}

The study was granted ethical clearance by the University of Botswana, Ministry of Health and PMH Institutional Review Boards. Written informed consent was obtained before data collection from all participants or their relatives in the cases where the patient was incapacitated to consent.

\section{Results}

In total, 400 patients with a median age of 35.5 (IQR 25-50) years were enrolled in the study. Demographic profile of the participants including their co-morbidities is shown in Table 1 . There were $143(35.8 \%)$ emergency and $257(64.2 \%)$ elective surgeries. The most common operations performed were explorative laparotomy (25\%), appendectomy (18.3\%), excisions and mastectomy (8\%). 
Table 1: Clinical and demographic characteristics of enrolled surgical patients at Princess Marina Hospital in Botswana, $\mathrm{N}=400$

\begin{tabular}{|c|c|c|c|c|}
\hline \multirow{2}{*}{\multicolumn{2}{|c|}{ Characteristics }} & \multirow{2}{*}{$\begin{array}{c}\text { Emergency } \\
143 \text { cases }\end{array}$} & \multirow{2}{*}{$\begin{array}{c}\text { Elective } \\
257 \text { cases }\end{array}$} & \multirow{3}{*}{$\begin{array}{c}\text { Total } \\
400 \text { cases } \\
\underline{209(52.3)} \\
\end{array}$} \\
\hline & & & & \\
\hline sex-n (\%) & Female & $50(35.0)$ & $159(61.9)$ & \\
\hline & $\underline{\text { Male }}$ & $90(62.9)$ & $98(38.1)$ & $191(49.7)$ \\
\hline \multicolumn{2}{|c|}{ Age - years, median (IQR) } & $43(29-55)$ & $26(22-36)$ & $35.5(25-50)$ \\
\hline \multirow{11}{*}{ Surgeries-n (\%) } & Explorative laparotomy & $\underline{74(51.7)}$ & 26(10.1) & $100(25.0)$ \\
\hline & Appendectomy & $\underline{66(46.2)}$ & $\underline{7(2.7)}$ & $73(18.3)$ \\
\hline & Excision & $\underline{1(0.7)}$ & $\underline{49(19.1)}$ & $50(12.5)$ \\
\hline & Mastectomy & $\underline{0}$ & $\underline{32(12.5)}$ & $\underline{32(8.0)}$ \\
\hline & Herniorraphy & $3(2.1)$ & $\underline{25(9.7)}$ & $\underline{28(7.0)}$ \\
\hline & Hydrocoelectomy & $\underline{2(1.4)}$ & $19(7.4)$ & $\underline{21(5.3)}$ \\
\hline & Cholecystectomy & $1(0.7)$ & $\underline{20(7.8)}$ & $\underline{21(5.3)}$ \\
\hline & Orchiectomy & $\underline{5(3.5)}$ & $\underline{12(4.7)}$ & $17(4.3)$ \\
\hline & Mammoplasty & $\underline{0}$ & $\underline{15(5.8)}$ & $\underline{15(3.8)}$ \\
\hline & Knee amputation & $\underline{0}$ & $12(4.7)$ & $12(3)$ \\
\hline & Others & $\underline{10(7.0)}$ & $44(17.1)$ & $\underline{54(11.3)}$ \\
\hline \multirow{5}{*}{ Co-morbidities-n (\%) } & $\underline{\text { HIV }}$ & $21(14.7)$ & $56(21.8)$ & $77(19.5)$ \\
\hline & Hypertension & $\underline{7(4.9)}$ & $55(21.4)$ & $\underline{62(15.6)}$ \\
\hline & Cancer & $\underline{0}$ & $\underline{41(16.0)}$ & $\underline{41(10.3)}$ \\
\hline & Tuberculosis & $\underline{2(1.4)}$ & $\underline{2(0.8)}$ & $\underline{4(1)}$ \\
\hline & Diabetes & $\underline{2(1.4)}$ & $\underline{20(7.8)}$ & $\underline{22(5.5)}$ \\
\hline
\end{tabular}

NB: IQR = Interquartile range

Antibiotic use

Overall, 293(73.3\%) cases were prescribed antibiotics. Sixty (15\%) patients received antibiotics preoperatively, $233(58.3 \%)$ patients post-surgery, and 107(26.8\%) patients were not prescribed any antibiotics (Table 2). 
Pre-operative antibiotics were prescribed in 31 (21.7\%) emergency surgeries as compared to 29 $(11.3 \%)$ elective surgeries $(P=0.005)$. Post-operative antibiotics were prescribed in $132(92.3 \%)$ emergency surgeries as compared to $144(56 \%)$ elective surgeries $(P<0.001)$. Of the patients who started antibiotics postoperatively, established infections was suspected in those with peritonitis (8 cases), abscesses (4 cases) and appendicitis (50 cases). The mean (SD) duration of post-operative antibiotic use was 5 (2.6) days. The most common prescribed antibiotics were cefotaxime $(80.7 \%)$, metronidazole $(63.5 \%)$, cefradine $(13.6 \%)$ and amoxicillin/clavulanate $(11.6 \%)$. Co-administration of cefotaxime and metronidazole was found in $134(48.6 \%)$ patients post-operatively. Oral antibiotics such as cloxacillin, amoxicillin, doxycycline and metronidazole were also prescribed.

Table 2: Antibiotic prophylaxis among surgical patients at Princess Marina Hospital in Botswana.

All Pre-operative $\quad$ Post-operative

\begin{tabular}{|c|c|c|c|c|c|}
\hline Antibiotics - & & & & & \\
\hline - & & Emergency & Elective & Emergency & Elective \\
\hline & 293 cases & 31 cases & 29 cases & 104 cases & 129 cases \\
\hline Cefotaxime & $220(80.7)$ & 17(54.8) & 16(55.2) & 88(84.6) & 99(76.7) \\
\hline Metronidazole & $\underline{186(63.5)}$ & $15(48.4)$ & $\underline{6(20.7)}$ & $90(86.5)$ & 75(58.1) \\
\hline Cefradine & $\underline{40(13.6)}$ & $8(25.8)$ & $13(44.8)$ & $\underline{11(10.6)}$ & 8(6.2) \\
\hline Amoclav & $\underline{34(11.6)}$ & $\underline{0}$ & $\underline{0}$ & 14(13.5) & $20(15.5)$ \\
\hline Amoxicillin & $\underline{17(5.8)}$ & $\underline{0}$ & $\underline{0}$ & $\underline{2(1.9)}$ & 15(11.6) \\
\hline Gentamicin & $\underline{14(4.8)}$ & $1(3.2)$ & $\underline{0}$ & $\underline{11(10.6)}$ & $2(1.6)$ \\
\hline Tazocin & $\underline{9(3.1)}$ & $\underline{0}$ & $\underline{0}$ & $4(3.8)$ & $5(3.9)$ \\
\hline Cefriaxone & $\underline{3(1.0)}$ & $1(3.2)$ & $\underline{0}$ & $\underline{0(1.9)}$ & $2(1.6)$ \\
\hline
\end{tabular}

NB: Amoclav = amoxicillin- clavulanic

\section{Surgical site infections}

Fifty two (13\%) patients self-reported purulent discharge from operation sites. The diagnosis of an SSI was made by the doctor in $36(9 \%)$ patients and positive cultures were reported in $20(5 \%)$ patients. The rate of SSI among patients who underwent emergency and elective surgeries were $22(8.5 \%)$ and $14(9.9 \%)$ respectively. Culture positive SSI occurred in $11(55 \%)$ emergency and in $9(45 \%)$ elective operations. The majority $(88.2 \%)$ of patients with culture positive SSI had not received preoperative antibiotics. Two thirds (65\%) of the patients were women. Three of the patients who had positive culture results were HIV positive.

The most common types of organisms isolated from the SSIs are shown in figure 1. Staphylococcus aureus, Pseudomonas auriginosa and Escherichia coli were the most common isolated pathogens. All these organisms were sensitive to cefotaxime, the most commonly used antibiotic. Escherichia coli, Klebsiella spp. and Citrobacter spp., were however resistant to cefotaxime. There was no sensitivity testing for metronidazole or cefradine. 


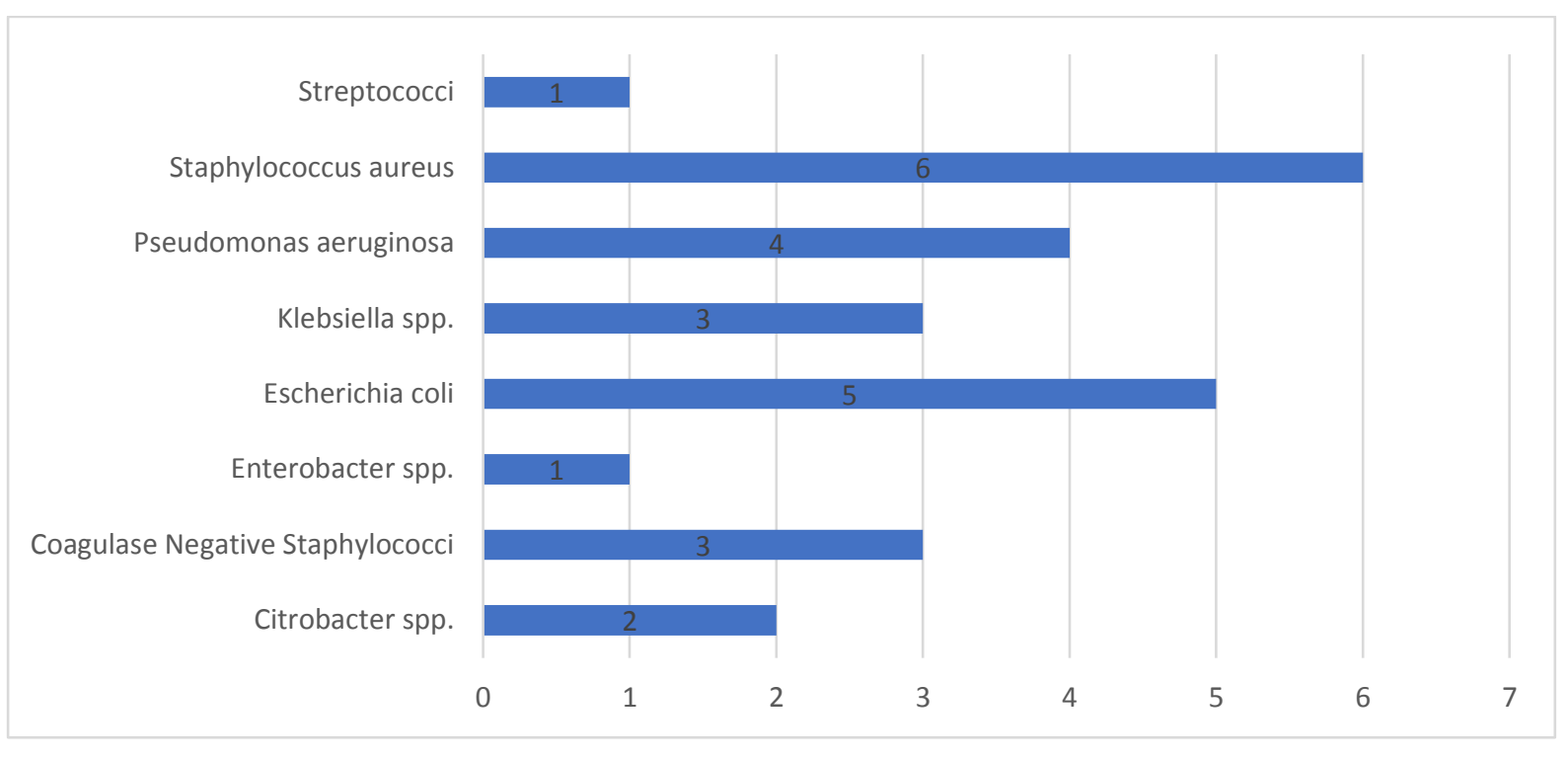

\section{Discussion}

In this study, we found that approximately three-quarters $(73 \%)$ of the patients received antibiotics peri-operatively, mostly after surgery. In addition, the majority of patients who had pre-operative antibiotics continued them post-operatively. The most frequently prescribed antibiotics were intravenous cefotaxime, cefradine and metronidazole. The incidence of SSIs was $8.8 \%$, and about half $(48.6 \%)$ of the patients with SSI had culture positive pus swabs.

The SSI rate in this hospital in Botswana is lower than the pooled incidence rate of SSI of $14.8 \%$ reported in Africa by Andersen et al [5]. It is, however, comparable to the incidence rate of $9.2 \%$ reported by Ntsama et al among surgical patients in three public hospitals in Yaounde [35]. While the SSI rate in Botswana is also lower than in other developing countries, where rates as high as $41 \%$ have been reported, it is higher than findings from developed countries where it is reported $0-3 \%$ of surgical patients develop SSIs [7-9]. Nevertheless, it is difficult to compare incidence rates across countries due to variations in populations including co-morbidities, types of surgery, hospital sizes, experience of the surgeons and surveillance methods [3, 4]. One of the reasons for the apparent lower rate of SSIs in Botswana could be the high uptake of antibiotics peri-operatively. The use of antibiotics is reported to decrease the incidence of SSI by $39 \%$, primarily in those patients who receive antibiotics pre-operatively $[23,26]$. However, as the majority of our patients received antibiotics post-operatively in our study, the lower incidence of SSIs cannot fully be explained by antibiotic use.

Similar to findings from other studies, the most common organisms in the current study were Pseudomonas aeruginosa, Staphylococcus aureus, and coagulase-negative staphylococci [36-38]. Generally, the causative pathogens for SSI originate from the patient's endogenous flora and depend on the type and site of surgery [38].

In addition to other measures, the World Health Organisation guidelines for the prevention of SSIs emphasize an optimal timing for preoperative antimicrobial prophylaxis when indicated [20]. In this study, we found that the timing of the initiation of antibiotics, antibiotic selection and duration of antibiotic prophylaxis were not according to the published guidelines $[2,13,26]$. The finding is consistent with previous studies which revealed heterogeneity in antibiotic choices, timing and duration of antibiotic prophylaxis among surgical patients [39]. To prevent SSIs, the guidelines advise that antibiotic prophylaxis should be given 1-2 hours before a surgical incision for adequate serum and tissue drug levels to be achieved during surgery [13, 17, 22, 24-26]. Similar to findings by Gans et al. where $59 \%$ of patients in selected American hospitals continued antibiotics postoperatively, the majority of our patients received antibiotics after surgery [39]. Largely, the majority of our patients were administered antibiotics for a mean duration of 5 days. This is contrary to the evidence showing 
that the administration of antibiotics after the incision is associated with an increased predisposition to antibiotic resistance and hospital costs compared with administration of antibiotics prior to the incision $[20,29]$. In actual fact, post-operative initiation of antibiotics has the same effect on preventing SSI as placebo $[29,40]$. For reasons which could not be ascertained in this study, we also found that most of those patients who received pre-operative antibiotics continued with them postoperatively for an average of five days. As mentioned above, postoperative antibiotic administration is no better than the preoperative administration alone and may enhance resistance rates $[20,29,41]$. Pre-operative use of antibiotics should therefore be encouraged in Botswana and we are now encouraging this in our hospital. This will be the subject of future research.

Normally, selection of antibiotic prophylaxis should be based on multiple factors including the normal floral distribution of the site to be operated and the antibiotic resistance patterns in the hospital setting $[1,17,27,31,32]$. Usually, broad-spectrum antibiotics, especially cephalosporin, are preferred to narrow spectrum antibiotics [27]. Because of its longer half-life than other first-generation cephalosporin, a single dose of cefazolin has been ideal for many procedures [42]. As cefazolin is not available in our setting, the Botswana national guidelines recommend cefradine, a first-generation cephalosporin, for most procedures [33]. Despite this, cefradine was prescribed in only $13 \%$ of all patients who were administered antibiotics. The majority of our patients received cefotaxime, often in combination with metronidazole. This low compliance with the guideline not only increases hospital costs but also poses an increased risk of resistance to cefotaxime, increasing the occurrence of antibiotic resistance which we need to address [2].

\section{Limitations}

We are aware that our study was only undertaken in one hospital in Botswana. However, as mentioned, this is a leading tertiary hospital in the country; consequently, a similar or worse picture is likely to be seen in secondary hospitals in Botswana. Although the exclusion of patients with prosthetic implants may have underestimated the incidence of SSI in our study, we included most of the surgeries encountered in most general hospitals in Botswana for comparative purposes. Even so, surgical implantation of prostheses is not a common practice at PMH. Another limitation of the study was the reliance on surgeon's and anaesthetist's notes as evidence for pre-operative and intraoperative use of antibiotics. This is always a concern with such a methodology. Finally, we did not break SSI down into superficial, deep and organ/space related, and were unable to discuss the associated clinical consequences. Nevertheless, in view of our extensive methodology, we believe our findings are robust and provide guidance to this and other hospitals in Botswana as we seek to enhance the appropriate use of antibiotics in our country.

\section{Conclusion}

In conclusion, whilst our rate of SSIs at $8.8 \%$ appears lower than in other African countries, there are considerable areas for improvement to reduce the incidence of SSIs and potential AMR in Botswana. These include the timing of the prophylaxis, choice of antibiotics, and duration of prophylaxis beyond surgery. These findings call for interventions to improve the rational use of antibiotic prophylaxis to decrease the rate of SSIs in Botswana. We have already started these programmes in our hospital to address the concerns, and we will be following this up in future research projects. Research projects are also starting at other hospitals in Botswana building on these findings.

\section{Key points}

- Appropriate antibiotic prophylaxis is known to reduce the incidence of surgical site infections (SSIs), shorten hospital stay and reduce costs

- Current guidelines recommend that the first dose of antibiotic prophylaxis should be given 1-2 hours before the first incision and not after the first incision or post operatively for maximum impact to reduce SSIs and potentially resistance rates. To maintain adequate drug concentration at surgical site, re-dosing is advised for surgeries that exceed 2 half-lives of the antimicrobial or if there is excessive blood loss. However, there is variation in the use of prophylactic antibiotics across countries, with the need for prophylaxis greater in lower and middle income countries with more complex cases

- SSIs in this leading tertiary hospital in Botswana following antibiotic use and follow-up of patients up to 30 days post operatively was $8.8 \%$ 
- Antibiotics were given in $73.3 \%$ of patients undergoing surgery, principally post-operatively $(58.3 \%)$ with cefotaxime and metronidazole the most prescribed antibiotics

- Concerns that the low compliance with Botswana guidelines will increase costs as well as increase resistance rates. These concerns are already resulting in interventions to try and address this in this and other hospitals in Botswana, which is being followed up

Competing interest and acknowledgements.

The authors declare no competing interests with this study. There was no external funding for this study. However, BBG received a small grant from the Karolinska to support the write-up of the paper.

\section{Author contributions}

JCM, SS, MGMDM and MM designed the study and were principally involved in data collection and analysis. JCM, SS, AM and BG produced the initial draft manuscript. All authors critiqued successive drafts of the manuscript before submission.

\section{References}

1. Horan TC, Gaynes RP, Martone WJ, Jarvis WR and Emori TG. CDC definitions of nosocomial surgical site infections, 1992: a modification of CDC definitions of surgical wound infections. Infection control and hospital epidemiology. 1992; 13: 606-8.

2. Weber WP, Marti WR, Zwahlen M, Misteli H, Rosenthal R, Reck S, et al. The timing of surgical antimicrobial prophylaxis. Ann Surg. 2008;247(6):918-26.

3. Young PY, Khadaroo RG. Surgical site infections. The Surgical clinics of North America. 2014; 94: $1245-64$.

4. Jenks $P$, Laurent $M$, McQuarry $S$, Watkins R. Clinical and economic burden of surgical site infection (SSI) and predicted financial consequences of elimination of SSI from an English hospital. Journal of Hospital infection. 2014; 86: 24-33.

5. Anderson DJ, Podgorny K, Berrios-Torres SI, et al. Strategies to prevent surgical site infections in acute care hospitals: 2014 update. Infect Control Hosp Epidemiol. 2014; 35: 605-27.

6. Najjar PA, Smink DS. Prophylactic antibiotics and prevention of surgical site infections. The Surgical clinics of North America. 2015; 95: 269-83.

7. Nejad SB, Allegranzi B, Syed SB, Ellis B and Pittet D. Health-care-associated infection in Africa: a systematic review. Bulletin of the World Health Organization. 2011; 89: 757-65.

8. Mawalla B, Mshana S, Chalya PL. Imirzalioglu C. Mahalu W. Predictors of surgical site infections among patients undergoing major surgery at Bugando Medical Centre in Northwestern Tanzania. BMC Surgery. 2011; 11.

9. Allegranzi B, Nejad SB, Combescure C, et al. Burden of endemic health-care-associated infection in developing countries: systematic review and meta-analysis. The Lancet. 2011; 377: 228-41. 10. Korol E, Johnston $\mathrm{K}$, Waser $\mathrm{N}$, et al. A systematic review of risk factors associated with surgical site infections among surgical patients. PloS one. 2013; 8: e83743.

11. Ngaroua, Ngah J, Bénet T and Djibrilla Y. Incidence of surgical site infections in sub-Saharan Africa: systematic review and meta-analysis. The Pan African medical journal. 2015; 24: 171 -.

12. Cai LZ, Chang J, Weiser TG, Forrester JD. Surgical Site Infections after Tissue Flaps Performed in Low- and Middle-Human Development Index Countries: A Systematic Review. Surgical infections. 2017; 18: 765-73.

13. Woods RK, Dellinger EP. Current guidelines for antibiotic prophylaxis of surgical wounds. American Family Physician. 1998; 57: 2731.

14. Lilani S, Jangale N, Chowdhary A, Daver G. Surgical site infection in clean and cleancontaminated cases. Indian journal of medical microbiology. 2005; 23: 249.

15. Eriksen HM, Chugulu S, Kondo, Lingaas E. Surgical-site infections at Kilimanjaro Christian Medical Center. Journal of Hospital Infection. 2003; 55: 14-20.

16. Santos K, Fonseca L, Bravo Neto G, Gontijo Filho P. Surgical site infection: rates, etiology and resistance patterns to antimicrobials among strains isolated at Rio de Janeiro University Hospital. Infection. 1997; 25: 217-20.

17. Bratzler DW and Houck PM. Antimicrobial prophylaxis for surgery: an advisory statement from the National Surgical Infection Prevention Project. Clinical Infectious Diseases. 2004; 38: 1706-15.

18. Stanic S, Bojanic J, Grubor P, Mijovic B, Maric V. Examination of Risk Factors for the Development of Surgical Site Infections. Materia socio-medica. 2017; 29: 134-7. 
19. Testa M, Stillo M, Giacomelli S, et al. Appropriate use of antimicrobial prophylaxis: an observational study in 21 surgical wards. BMC Surg. 2015; 15: 63.

20. WHO. GLOBAL GUIDELINES FOR THE PREVENTION OF SURGICAL SITE INFECTION. 2016. Availble at URL: http://www.who.int/gpsc/global-guidelines-web.pdf.

21. Bratzler DW and Hunt DR. The surgical infection prevention and surgical care improvement projects: national initiatives to improve outcomes for patients having surgery. Clinical infectious diseases. 2006; 43: 322-30.

22. Antibiotic prophylaxis in surgery. A national clinical guideline. SIGN. NHS Quality Improvement Scotland (2008). Available at URL: http://www.just.edu.jo/ar/DIC/ClinicGuidlines/Antibiotic\%20prophylaxis\%20in\%20surgery.pdf

23. Smaill FM, Gyte GM. Antibiotic prophylaxis versus no prophylaxis for preventing infection after cesarean section. Cochrane Database Syst Rev. 2010 Jan 20;(1):CD007482

24. Dellinger EP. What is the ideal time for administration of antimicrobial prophylaxis for a surgical procedure? Ann Surg. 2008;247(6):927-8

25. Ban KA, Minei JP, Laronga C, et al. Executive Summary of the American College of Surgeons/Surgical Infection Society Surgical Site Infection Guidelines-2016 Update. Surgical infections. 2017; 18: 379-82.

26. de Jonge SW, Gans SL, Atema JJ, Solomkin JS, Dellinger PE and Boermeester MA. Timing of preoperative antibiotic prophylaxis in 54,552 patients and the risk of surgical site infection: A systematic review and meta-analysis. Medicine. 2017; 96: e6903.

27. Bratzler DW, Dellinger EP, Olsen KM, et al. Clinical practice guidelines for antimicrobial prophylaxis in surgery. Surgical infections. 2013; 14: 73-156.

28. de Jonge SW, Gans SL, Atema JJ, Solomkin JS, Dellinger PE and Boermeester MA. Timing of preoperative antibiotic prophylaxis in 54,552 patients and the risk of surgical site infection: A systematic review and meta-analysis. Medicine. 2017; 96.

29. NICE. Surgical site infection, prevention and treatment. 2008. Available at URL: https://www.nice.org.uk/guidance/cg74

30. Worth LJ, Bull AL, Spelman T, Brett J, Richards MJ. Diminishing surgical site infections in Australia: time trends in infection rates, pathogens and antimicrobial resistance using a comprehensive Victorian surveillance program, 2002-2013. Infect Control Hosp Epidemiol. 2015; 36: 409-16.

31. Culver DH, Horan TC, Gaynes RP, Martone WJ, Jarvis WR, Emori TG, et al . Surgical wound infection rates by wound class, operative procedure and patient risk index. National Nosocomial Infection surveillance System. Am J Med. 1991; 91.

32. Cruse PJ, Foord R. The epidemiology of wound infection.A 10-year prospective study of 62939 wounds. The Surgical clinics of North America. 1980; 60: 27-40.

33. BEDAP. Botswana Antimicrobial Therapy Guide. NASCOD. 2 ed. Botswana: Ministry of Health, 2012, p. 136-52.

34. AVERT. HIV and AIDS in Botswana. Available at URL: https://www.avert.org/professionals/hivaround-world/sub-saharan-africa/botswana

35. Ntsama E, Avomo J, Esiene A, et al. Prevalence of surgical site infections and evaluation of risk factors after surgery, case of three public hospitals in Cameroon. Journal of Medicine and Medical Sciences. 2013; 4: 241-6.

36. Fan $\mathrm{Y}$, Wei $\mathrm{Z}$, Wang $\mathrm{W}$, et al. The incidence and distribution of surgical site infection in mainland China: a meta-analysis of 84 prospective observational studies. Scientific reports. 2014; 4.

37. Eriksen $\mathrm{H}$, Chugulu S, Kondo $\mathrm{S}$ and Lingaas E. Surgical-site infections at Kilimanjaro Christian medical center. Journal of Hospital Infection. 2003; 55: 14-20.

38. Mangram AJ, Horan TC, Pearson ML, Silver LC, Jarvis WR and Committee HICPA. Guideline for prevention of surgical site infection, 1999. American journal of infection control. 1999; 27: 97-134.

39. Gans I, Jain A, Sirisreetreerux N, Haut ER, Hasenboehler EA. Current practice of antibiotic prophylaxis for surgical fixation of closed long bone fractures: a survey of 297 members of the Orthopaedic Trauma Association. Patient safety in surgery. 2017; 11: 2.

40. Stone HH, Hooper CA, Kolb LD, Geheber CE and Dawkins EJ. Antibiotic prophylaxis in gastric, biliary and colonic surgery. Annals of surgery. 1976; 184: 443.

41. Stone HH, Haney BB, Kolb LD, Geheber CE, Hooper CA. Prophylactic and preventive antibiotic therapy: timing, duration and economics. Annals of surgery. 1979; 189: 691.

42. Dimick JB, Lipsett PA, Kostuik JP. Spine update: antimicrobial prophylaxis in spine surgery: basic principles and recent advances. Spine. 2000; 25: 2544-8. 